\title{
COSTOS INTERNACIONALES PARA IMPORTAR Y EXPORTAR
}

\author{
INTERNATIONAL COSTS TO IMPORT AND EXPORT \\ Agustín Rodríguez Chavez* \\ Docente Asociado de la Facultad de Ciencias Contables \\ Universidad Nacional Mayor de San Marcos-UNMSM / Lima-Perú \\ [Recepción: Abril 2016/ Conformidad: Mayo 2016]
}

\section{RESUMEN}

Los cambios experimentados constantemente por los procesos de negocios internacionales se originan en la variación de las políticas de los países, el uso de nuevas tecnologías o la implementación de estrategias corporativas de negocios frente a los operadores logísticos y las fluctuaciones de la economía. Debido a ello, en la actualidad es importante estudiar y analizar los costos asociados a las importaciones y exportaciones.

En el año 2010 el Perú importaba 21 mil millones de dólares y exportaba 13 mil millones de dólares. Luego, en el año 2015 hemos pasado a importar 14 mil millones de dólares y exportar 27 mil millones de dólares. Este cambio nos lleva a proponer estructuras que incluyan los costos asociados a los procesos de importaciones y exportación.

Hoy es necesario monitorear el comportamiento de los costos diferenciando los costos de adquisición, aplicando la NIC 2 Inventarios, evaluando los costos directos e indirectos del proceso y cuándo estos se deben integrar o excluir del costo de adquisición. Las diferencias de los tipos de cambios de las monedas asociadas al proceso nos conducen a analizar el impacto sobre los costos, considerando, además, que, de acuerdo a la NIC 21 Efectos de las variaciones en las tasas de cambio de la moneda extranjera, las diferencias de cambio no forman parte del costo de adquisición, en especial, si están asociados al costo total para la determinación de precios en moneda extranjera.

Palabras Clave:

NIC 2; NIC21; costo de importación y exportación; moneda extranjera; proceso de importación y exportación; drawback.

\begin{abstract}
The constantly experienced changes by the international business processes are caused policy changes of the countries, the new technologies or the implementation of corporative business strategies facing logistics operators and fluctuations of economy. For this reason, in the present time it important to study and analyze the costs associated with imports and exports.

In 2010, Peru imported 21 billion dollars and exported 13 billion dollars. Then, in 2015 we imported 14 billion dollars and exported 27 billion dollars. This change leads us to propose structures which reflect the costs associated with the processes import and export. Today we must monitor the behavior of costs differentiating them from acquisition costs in accordance with IAS 2 Inventories, evaluating direct and indirect costs of the process as these should be integrated or excluded from the cost of acquisition.

The differences in exchange rates of currencies associated with the process, forces us to analyze the impact on cost considering also that according to IAS 21 The Effects of Changes in Exchange Rate of Foreign Exchange, the exchange differences are not part of the acquisition cost, specially, if the total costs are associated with pricing in foreign currency.
\end{abstract}

\section{Keywords:}

IAS 2; IAS 21; cost of import and export; foreign exchange; import and export process; drawback.

\footnotetext{
* Magíster en Costos y Presupuestos. Contador público colegiado y certificado - UNMSM. Email: arodriguezc@unmsm.edu.pe.

** Colaboradora: Ingrid Rodríguez Castro. Contadora pública - UNMSM.
} 


\section{SISTEMA DE COSTOS INTERNACIONALES}

Los incrementos en los niveles de importación y exportación de la última década nos señalan la necesidad de revisar la vigencia de los modelos y estructuras de costos aplicables. En el año 2007 importábamos 14 mil millones de dólares y exportábamos $12 \mathrm{mil}$ millones de dólares. En contraste, en el año 2015 hemos importado 14 mil millones de dólares y exportado 27 mil millones de dólares. En ese contexto, el comportamiento global de las economías frente al valor de su moneda nos conduce a analizar su impacto en los costos de nuestras operaciones internacionales. A manera de ejemplo, cabe comentar que un gerente cuya empresa debe 30 mil euros requiere de $44 \mathrm{mil}$ dólares si desea saldarla estando en el Perú. No obstante, si este viaja con los 44 mil a Miami para realizar dicho trámite no solo pagaría la deuda y cubriría sus gastos de viaje, sino que además le quedaría un saldo. Por otro lado, si, por ejemplo, alguien quiere importar un repuesto y lo solicita a la representante de la casa comercial en Miami, este producto se fabricará en Corea, se embarcará desde Tailandia y el pago final lo recibirá la filial de Panamá.

\section{LOS COSTOS Y SU INCIDENCIA EN LOS PROCESOS DE IMPORTACIÓN}

Para una adecuada definición de la estructura de costos del proceso de importación debemos de partir de la elaboración de un flujo que incluya las actividades asociadas al proceso.

A partir de este esquema se podrán identificar:

- Los costos de adquisición.

- Los costos directos del proceso de importar.

- Los costos indirectos del proceso de importar.

En el análisis de los costos se debe tomar en cuenta lo que hay que incluir en el costo de adquisición o considerar como parte del costo del proceso de importación. En el caso de una importación de materia prima el costo no asociado a la adquisición será el costo indirecto de producción, mientras que en el caso de la mercadería será parte de los gastos de administración, si la logística está dentro de esta función.

En adelante, tomaremos como referencia a una operación de importación de planchas de melanina de Canadá hecha mediante el régimen de importación para el consumo.

\section{COSTO DE ADQUISICIÓN}

Estos son los costos directos del proceso y que forman parte del costo del bien importado (objeto de costos). El costo de adquisición de los bienes importados incluye el precio de compra (sin considerar el IGV), los aranceles de importación, otros impuestos que no sean recuperables posteriormente de las autoridades fiscales, el pago de seguros y transportes, $\mathrm{y}$ otros costos directamente atribuibles a la adquisición de mercaderías, materiales y servicios. Los descuentos comerciales, las rebajas y otras partidas similares se deben deducir para determinar el costo de adquisición.

1. Precio de compra: Este se halla incluido en el invoice (factura) según el Incoterm pactado con el proveedor del exterior. Los más usuales son:

-FOB: Mediante este el proveedor asume los costos y responsabilidades hasta que la mercadería se encuentre a bordo del barco.

- CFR: Mediante este el proveedor asume los costos hasta el momento del transporte de la mercadería.

- CIF: Mediante este el proveedor asume los costos y responsabilidades hasta el transporte de la mercadería, lo que incluye el seguro.

En el modelo propuesto Forestal Rosa S.A.C.importa de Canadá planchas de melanina y el precio es fijado a FOB por cada plancha, de tal manera que se recibe un invoice por U\$30 000.

2. Flete del exterior: En una importación a precio FOB el importador asume los costos del flete del exterior. Para asignar los costos se debe verificar el contrato sobre el cual se está pagando dicho flete, puede ser por peso, por volumen, por contenedor o por valor; es sobre esa base contratada es que se asignan los costos a cada artículo importado.

En el modelo planteado, se paga el flete marítimo de Canadá al Perú en base al peso en kilos de melanina y se consigna el pago en la guía por U $\$ 6800$. Así, tendríamos la distribución de \$6800/34000 kilos $=0.20 \mathrm{x}$ kilo. 
3. Seguro: En una importación a precio FOB el importador asume los costos del seguro desde el exterior hasta el ingreso a su almacén. Para asignar estos costos se debe verificar el contrato sobre el cual se está pagando dicho seguro. Usualmente, se paga una prima sobre el FOB y es sobre esa base contratada que se asignan los costos a cada artículo importado. Por otro lado, existen casos en los que la empresa no toma el seguro.

En el modelo planteado, se paga el seguro de Canadá al Perú en base $0.60 \%$ del valor del FOB y se recibe la factura o aplicación de la prima por U\$ 180. Así, tendríamos la distribución de $\$ 180 / \$ 30$ $000=\$ 0.006 \times$ cada $\$$ de FOB.

4. Derechos aduaneros: Los pagos obligatorios se calculan sobre la base del CIF (FOB + flete + seguro). La Sunad incluirá un valor de seguro aun cuando la empresa no lo hubiera tomado. Para asignar los costos se debe verificar el porcentaje del arancel $(\mathrm{AV}=\mathrm{ad} /$ valorem $)$ de la declaración del DAM (DUA). La Sunad puede considerar un mayor valor para el cálculo. Por su parte, el artículo específico puede estar sujeto a convenios de disminución de AV.

En el modelo planteado, se paga por AV el $12 \%$ por cada artículo. Esto permite el siguiente cálculo: $12 \%$ x $36800=\$ 6800$.

5. Servicio de agente: En una importación es usual el cobro sobre un valor de referencia como el FOB, el total del valor administrado (incluyendo impuestos) o un valor fijo. A partir de ello se asignan los costos a cada artículo importado.

En el modelo planteado se paga los servicios de agente de aduana en base al $2 \%$ del valor FOB y se recibe la factura por $\$ 600$. Así, tendríamos una distribución de $2 \%$ en relación a $\$ 15000=\$ 300$ $\mathrm{x}$ cada artículo.

Tabla No 01:

Invoice mercadería

\begin{tabular}{|l|c|c|c|c|c|c|}
\hline \multirow{2}{*}{ INVOICE } & \multicolumn{7}{|c|}{ IMPORTACIÓN EN DÓLARES } \\
\cline { 2 - 7 } & Fecha & Base & $\begin{array}{c}\text { Laminado } \\
\text { QA }\end{array}$ & $\begin{array}{c}\text { Laminado } \\
\text { XA }\end{array}$ & Total & \\
\hline Planchas & & & 600 & 400 & $1.000,00$ & Unidades \\
\hline Peso & & Fact & 18000 & 16000 & $34.000,00$ & Kls \\
\hline Precio Unitario & & & $\$ 25,00$ & $\$ 37,50$ & & \\
\hline FOB & $28 / 01 / 16$ & Fact & $15.000,00$ & $15.000,00$ & $30.000,00$ & $100,0 \%$ \\
\hline
\end{tabular}

Fuente: Elaboración propia.

Tabla No 02:

Consignación del flete

\begin{tabular}{|l|c|c|c|c|c|c|}
\hline \multirow{3}{*}{ FLETE } & \multicolumn{6}{|c|}{ IMPORTACIÓN EN DÓLARES } \\
\cline { 2 - 7 } & Fecha & Base & $\begin{array}{c}\text { Laminado } \\
\text { QA }\end{array}$ & $\begin{array}{c}\text { Laminado } \\
\text { XA }\end{array}$ & Total & \\
\hline Planchas & & & 600 & 400 & $1.000,00$ & Unidades \\
\hline Peso & & Fact & 18000 & 16000 & $34.000,00$ & KLS \\
\hline Precio Unitario & & & $\$ 25,00$ & $\$ 37,50$ & & \\
\hline FOB & $28 / 01 / 16$ & Fact & $15.000,00$ & $15.000,00$ & $30.000,00$ & $100,0 \%$ \\
\hline Flete & $9 / 02 / 16$ & Peso & $3.600,00$ & $3.200,00$ & $6.800,00$ & $22,7 \%$ \\
\hline
\end{tabular}

Fuente: Elaboración propia. 
Tabla No 03:

Consignación del seguro

\begin{tabular}{|l|c|c|c|c|c|c|}
\hline \multirow{2}{*}{$\begin{array}{l}\text { FLETE } \\
\text { EXTERIOR }\end{array}$} & \multicolumn{6}{|c|}{ IMPORTACIÓN EN DÓLARES } \\
\cline { 2 - 7 } & Fecha & Base & $\begin{array}{c}\text { Laminado } \\
\text { QA }\end{array}$ & $\begin{array}{c}\text { Laminado } \\
\text { XA }\end{array}$ & Total & \\
\hline Planchas & & & 600 & 400 & $1.000,00$ & Unidades \\
\hline Peso & & Fact & 18000 & 16000 & $34.000,00$ & KLS \\
\hline Precio Unitario & & & $\$ 25,00$ & $\$ 37,50$ & & Dólares \\
\hline FOB & $28 / 01 / 16$ & Fact & $15.000,00$ & $15.000,00$ & $30.000,00$ & $100,0 \%$ \\
\hline Flete & $9 / 02 / 16$ & Peso & $3.600,00$ & $3.200,00$ & $6.800,00$ & $22,7 \%$ \\
\hline Seguro & $05 / 02 / 16$ & FOB & 90.00 & 90.00 & 180.00 & $0,60 \%$ \\
\hline
\end{tabular}

Fuente: Elaboración propia.

Tabla No 04:

Consignación de los derechos aduaneros

\begin{tabular}{|l|c|c|c|c|c|c|}
\hline \multirow{2}{*}{$\begin{array}{l}\text { FLETE } \\
\text { EXTERIOR }\end{array}$} & \multicolumn{7}{|c|}{ IMPORTACIÓN EN DÓLARES } \\
\cline { 2 - 7 } & Fecha & Base & $\begin{array}{c}\text { Laminado } \\
\text { QA }\end{array}$ & $\begin{array}{c}\text { Laminado } \\
\text { XA }\end{array}$ & Total & \\
\hline Planchas & & & 600 & 400 & $1.000,00$ & Unidades \\
\hline Peso & & Fact & 18000 & 16000 & $34.000,00$ & KLS \\
\hline Precio Unitario & & & $\$ 25,00$ & $\$ 37,50$ & & Dólares \\
\hline FOB & $28 / 01 / 16$ & Fact & $15.000,00$ & $15.000,00$ & $30.000,00$ & $100,0 \%$ \\
\hline Flete & $9 / 02 / 16$ & Peso & $3.600,00$ & $3.200,00$ & $6.800,00$ & $22,7 \%$ \\
\hline Seguro & $05 / 02 / 16$ & FOB & 90.00 & 90.00 & 180.00 & $0,60 \%$ \\
\hline Otros & & & & & 0,00 & \\
\hline CIF & & & $18.690,00$ & $18.290,00$ & $36.980,00$ & \\
\hline CIFDUA & $16 / 03 / 16$ & & $18.690,00$ & $18.290,00$ & $36.980,00$ & \\
\hline DERECHOS & & CIFDUA & $2.242,80$ & $2.194,80$ & $4.437,60$ & $12,00 \%$ \\
\hline
\end{tabular}

Fuente: Elaboración propia.

Tabla No 05:

Consignación de los servicios de agente

\begin{tabular}{|l|c|c|c|c|c|c|}
\hline \multirow{2}{*}{$\begin{array}{l}\text { FLETE } \\
\text { EXTERIOR }\end{array}$} & \multicolumn{7}{|c|}{ IMPORTACIÓN EN DÓLARES } \\
\cline { 2 - 7 } & Fecha & Base & $\begin{array}{c}\text { Laminado } \\
\text { QA }\end{array}$ & $\begin{array}{c}\text { Laminado } \\
\text { XA }\end{array}$ & Total & \\
\hline Planchas & & & 600 & 400 & $1.000,00$ & Unidades \\
\hline Peso & & Fact & 18000 & 16000 & $34.000,00$ & KLS \\
\hline Precio unitario & & & $\$ 25,00$ & $\$ 37,50$ & & Dólares \\
\hline FOB & $28 / 01 / 16$ & Fact & $15.000,00$ & $15.000,00$ & $30.000,00$ & $100,0 \%$ \\
\hline Otros internos & & & & & & \\
\hline Agencia Fact & $25 / 03 / 16$ & FOB & 300.00 & 300.00 & 600.00 & $2.00 \%$ \\
\hline
\end{tabular}

Fuente: Elaboración propia. 
6. Transporte, manipuleo interno: En una importación es usual el cobro por peso, por volumen, por contenedor o por valor, Para asignar los costos se debe verificar el contrato sobre el cual se está pagando dicho flete. Sobre esa base contratada se asignan los costos a cada artículo importado.

En el modelo planteado se paga el flete interno en base al precio de $S / 2.00$ por cada plancha. De ese modo, por 1000 unidades serían S/ 2000 al tipo de cambio de la fecha del transporte (27 de marzo de
2016). Tomando en cuenta el monto de $S / 3.403$ por cada $\$$, entonces, el flete calculado en dólares sería de 571.43.

Finalmente debemos puntualizar que en el costeo de una importación se deben incluir todos los costos directamente atribuibles. En ese sentido, si sumamos todos los elementos de los costos asignados por artículos obtendríamos el costo total y unitario por cada uno de ellos.

\section{Tabla No 06:}

Cálculo de precios en relación a la fecha de la operación

\begin{tabular}{|l|c|c|c|c|c|c|}
\hline \multirow{2}{*}{$\begin{array}{l}\text { FLETE } \\
\text { EXTERIOR }\end{array}$} & \multicolumn{6}{|c|}{ IMPORTACIÓN EN DÓLARES } \\
\cline { 2 - 7 } & Fecha & Base & $\begin{array}{c}\text { Laminado } \\
\text { QA }\end{array}$ & $\begin{array}{c}\text { Laminado } \\
\text { XA }\end{array}$ & Total & \\
\hline Planchas & & & 600 & 400 & $1.000,00$ & Unidades \\
\hline Peso & & Fact & 18000 & 16000 & $34.000,00$ & KLS \\
\hline Precio unitario & & & $\$ 25,00$ & $\$ 37,50$ & & Dólares \\
\hline Flete interno & $27 / 03 / 16$ & Unidad & 342.86 & 228.57 & 571.43 & $1.90 \%$ \\
\hline
\end{tabular}

Fuente: Elaboración propia. 


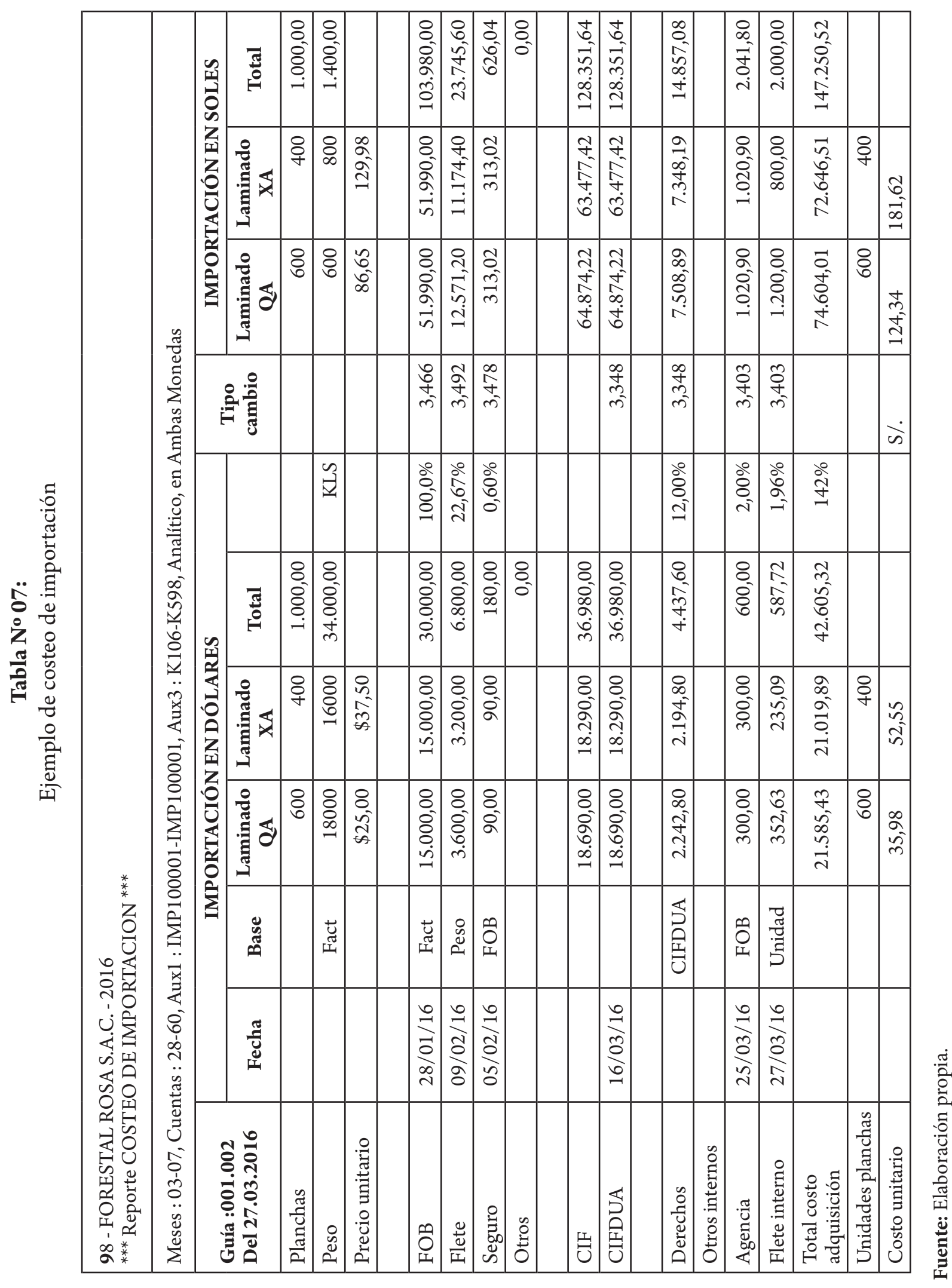




\section{IMPACTO DEL TIPO DE CAMBIO}

Hacer el seguimiento de las fechas de las operaciones es necesario para el cálculo del costo de la importación en soles. Así, podemos ver en nuestro modelo que el costo total de la importación en dólares alcanza los U\$ 42667.68 y en soles es de S/ 147 250.52. Dado que en nuestro país los precios deben ser publicados en soles, esa importación al tipo de cambio de febrero de 2016 (aproximadamente S/ 3.23 por dólar) sería de S/ 141577.47 , es decir, $9.6 \%$. A partir de este ejemplo, puede advertirse que es muy importante monitorear el comportamiento cambiario para incluirlo en nuestro modelo de formulación de los precios.

\section{COSTO DIRECTO DEL PROCESO DE IMPORTAR}

En las operaciones de importación se generan costos directos del proceso de importar pero que no podemos atribuir directamente al costo de adquisición, tales como las remuneraciones, las tecnologías yla infraestructura del área funcional responsable de la operación de importación. No obstante, esto es algo que no se debe pasar por alto. La razón es que si, por ejemplo, se elabora un presupuesto anual de importaciones de $\$ 480000$ anuales frente a los costos de la oficina de importación de $\$ 6000$ mensuales, esto nos arrojaría $\$ 72000$ anuales o $15 \%$ de costos sobre las importaciones hechas al año. A propósito de ello, vale mencionar que hoy existen operadores logísticos que, expertos en la gestión de importación, cobran entre $5 \%$ a $10 \%$ por cada operación. Una vez evaluado el costo obtenido de $15 \%$ frente al servicio de gestión e importación de 19\%, la gerencia podría decidir tercerizar el proceso y sería posible incluir ello en la estructura de adquisición.

\section{COSTO INDIRECTO DEL PROCESO DE MPORTAR}

Los costos indirectos son aquellos que no forman parte del costo de adquisición, ni se pueden asociar directamente al proceso de importar y que usualmente forman parte de la estructura de la empresa, tales como los costos de la gerencia de Logística y otros. Estos costos pueden ser tratados como gastos durante el periodo.

\section{LA FIJACIÓN DE LOS PRECIOS DE EXPORTACIÓN}

El estudio y análisis de costos para fijar precios de exportación se debe empezar identificando la estrategia de la cual la empresa está partiendo para desarrollar esta actividad. En este punto, hay dos escenarios posibles:

- Se está constituyendo una empresa exportadora. Este puede ser el caso de una empresa que esté exportando el $100 \%$ de la producción de espárrago o que planee vender muebles en el extranjero.

- Se está utilizando su capacidad marginal para exportar. El 70\% de la producción de cabinas de internet del mercado nacional quiere exportar con su capacidad marginal. En este caso, la Gerencia puede plantear una estrategia de exportación a costo marginal, es decir, aplicando los costos directos $y$ variables al producto exportable.

Una vez definido esto, la empresa debe hacer un análisis del mercado, según esté participando en:

- Un mercado con cotización internacional, como en el caso de la exportadora de espárragos.

- Ventas con mercados de precios libres, como en el caso de las ventas de cabinas de internet.

Después de efectuarse estas definiciones, la empresa debe desarrollar una estructura de costos separando sus componentes según su moneda de origen, ya sea en dólares (moneda extranjera) o en soles. Luego, la empresa debe revisar las normas para verificar si el producto tiene el beneficio de Drawback u otro. Para ello, debe analizar su estructura de costos para confirmar si tiene un:

- Componente importado por el cual pago de derechos aduaneros.

- Componente adquirido localmente para ver si la importación efectuada por su proveedor no tuvo beneficios sobre los derechos arancelarios.

\section{DRAWBACK}

Dice la Resolución del Tribunal Fiscal N²07901-2012:

Artículo $1^{\mathrm{o}}$.- Son beneficiarios del procedimiento de restitución simplificado de derechos arancelarios las empresas productoras-exportadoras, cuyo costo de producción haya sido incrementado por los derechos de aduana que 
gravan la importación de materias primas, insumos, productos intermedios y partes o piezas incorporados o consumidos en la producción del bien exportado, siempre que no exceda de los límites señalados en el presente Decreto.

En la página 54 de esta misma resolución, la administración señala que el $5 \%$ del valor de FOB, que representa lo efectivamente pagado por derechos arancelarios en la importación de materia prima incluida en el producto exportado, debe disminuir el costo de venta de la exportación por la cual se ha obtenido el drawback. Esto se sustenta en la NIC 2, que establece que el costo de compra de las importaciones efectuadas comprende los derechos de importación y otros impuestos, siempre que estos no sean recuperables por la empresa ante las autoridades fiscales.

De acuerdo a lo anteriormente propuesto, una estructura de ingresos y costos para una empresa que exporta a valor FOB debe incluir lo siguiente: 
Tabla No 08:

Estructura de ingresos y costos en exportacion de acuerdo a moneda de origen

\begin{tabular}{|c|c|c|c|c|c|c|}
\hline $\begin{array}{l}\text { Exportación de cabinas de internet en pallet } \\
\text { de } 10 \text { cabinas. Inicio } 10 \text { dic } 2015\end{array}$ & & Precio & $\begin{array}{l}\text { Total en } \\
\text { dólares }\end{array}$ & & $\begin{array}{c}\text { En } \\
\text { dólares }\end{array}$ & En soles \\
\hline VOLUMEN & & & 600 & ABINAS & & \\
\hline PRECIO / INGRESO FOB & $\mathrm{PR}$ & $\$ 172,00$ & $\$ 103.200,00$ & $100,0 \%$ & & \\
\hline Materiales Directos & & & $51.600,00$ & $-50,0 \%$ & $\$ 36.000,00$ & 53.040 \\
\hline Material Importado & MV & 60,00 & $36.000,00$ & $-34,9 \%$ & 36.000 & \\
\hline Material Nacional & MV & 5,00 & $3.000,00$ & $-2,9 \%$ & - & 10.200 \\
\hline Material Importado Proveedor Local & MV & 21,00 & $12.600,00$ & $-12,2 \%$ & & 42.840 \\
\hline Mano de Obra Directa & $\mathrm{MD}$ & 9,00 & $5.400,00$ & $-5,2 \%$ & - & 18.360 \\
\hline Servicios de Ensamblado & SV & 5,00 & $3.000,00$ & $-2,9 \%$ & - & 10.200 \\
\hline Servicio de empacado & SV & 2,00 & $1.200,00$ & $-1,2 \%$ & & 4.080 \\
\hline CIP Costo Ind.Prod. Exportable VARIABLE & IV & 5,00 & $3.000,00$ & $-2,9 \%$ & 2.000 & 3.400 \\
\hline CIP Costo Ind. Produc. Exportable FIJO & IF & 15,00 & $9.000,00$ & $-8,7 \%$ & - & 30.600 \\
\hline COSTO TOTAL & $\mathrm{CP}$ & & $73.200,00$ & $-70,9 \%$ & 36.000 & 116.280 \\
\hline Margen Bruto & & & $30.000,00$ & $29,1 \%$ & -36.000 & -116.280 \\
\hline Gastos Operativos de Exportación & GE & & $5.000,00$ & $-4,8 \%$ & 2.100 & 9.860 \\
\hline Embalado pallets & GV & 2,00 & $1.200,00$ & $-1,2 \%$ & & 4.080 \\
\hline Manipuleo & GV & 0,33 & 200,00 & $-0,2 \%$ & & 680 \\
\hline Transporte local & GV & & $2.000,00$ & $-1,9 \%$ & 2.000 & \\
\hline Seguro local & GV & 0,17 & 100,00 & $-0,1 \%$ & 100 & \\
\hline Gestion exportacion & GF & 1,67 & $1.000,00$ & $-1,0 \%$ & & 3.400 \\
\hline Promoción y Publicidad & GF & 0,83 & 500,00 & $-0,5 \%$ & & 1.700 \\
\hline Utilidad Operativa / Costo operativo & & & $25.000,00$ & $24,2 \%$ & -38.100 & -126.140 \\
\hline Ingreso por Drawback & DB & 4,53 & $2.718,00$ & $2,6 \%$ & - & 9.241 \\
\hline Gasto financiero asociado a la export. $3 \%$ & $\mathrm{FV}$ & $-3,91$ & $-2.346,00$ & $-2,3 \%$ & & -7.976 \\
\hline Impacto de la exposición cambiaria (3) & DC & 1,82 & $\$ 1.091,18$ & $1,1 \%$ & & \\
\hline Utilidad antes de Impuestos y Particip. & & & $26.463,18$ & $25,6 \%$ & -38.100 & -116.899 \\
\hline Participación de utilidades $\quad 10 \%$ & PU & $-4,41$ & $-2.646,32$ & $-2,6 \%$ & & -8.997 \\
\hline Utilidad antes de Impuestos & UT & $\$ 39,69$ & $\$ 23.816,86$ & $23,1 \%$ & $-\$ 38.100$ & -125.896 \\
\hline Costo operativo en dolares y soles & A & & & & $-\$ 38.100$ & $-\$ 37.100$ \\
\hline Determinación de Drawback (2) & $3 \%$ & $\$ 103.200$ & $-\$ 12.600$ & $=$ & $\$ 90.600$ & $\$ 2.718$ \\
\hline Tipo de cambio de exportación & Prof & orma $(\mathrm{P})$ & $\mathrm{S} / .3,400$ & $\mathrm{x} U \$$ & Cobranza & 3,500 \\
\hline \multicolumn{6}{|c|}{ (1)Operaciones en soles convertidos a dólares, según su fecha de operación. Promedio 2 meses } & $\mathrm{S} / .3,400$ \\
\hline \multicolumn{7}{|c|}{ (2) Para el Drawback no se incluye las compras locales de materiales importados, con beneficios aduaneros } \\
\hline \multicolumn{5}{|c|}{ (3) Diferencia de cambio favorable por los desembolsos operativos en soles } & $-\$ 37.100$ & $-\$ 1.091$ \\
\hline
\end{tabular}

Fuente: Elaboración propia. 
A partir de lo planteado se podrá obtener la estructura de precios siguiente:

1. Los costos de producción fijos y variables para analizar el comportamiento de los costos en base al volumen de producción exportable. Para un volumen de producción de 600 cabinas, el costo unitario de producción será de $\$ 122.00$. No obstante, si el volumen de producción fuera de 700 cabinas, el costo unitario sería cerca de $\$ 120$ (\$ $107+\$ 9000 / 700)$.

2. Los costos de distribución. Esto incluiría los costos de operación así como:

- Los costos de financiamientos asociados a la operación de exportación, los cuales, en el caso de la estructura planteada, incluyen un $3 \%$ sobre los costos de producción y costos operativos (3\% x (\$73200+\$5000)).

- El ingreso por drawback calculado para el año 2016 en 3\% sobre el valor FOB menos los costos de las compras locales de bienes importados que tuvieron beneficios en la importación. 3\% x $(\$ 103200-\$ 12600)=\$ 2718$. En este punto, se incluye la participación de utilidades considerando que este es un beneficio del trabajador, por lo que forma parte del costo de la mano de obra. Su cálculo es el $10 \%$ sobre la utilidad neta antes de aplicado el impuesto, o sea: $10 \%$ x 26 $463.18=\$ 2646.32$.

- El impacto favorable de la diferencia de cambio, el cual es originado por el incremento del tipo de cambio al momento de la cobranza (S/3.500 a febrero 2016) y el tipo de cambio promedio (S/ 3.40 promedio) utilizado en el pago de las operaciones en moneda nacional (esto equivaldría a S/ 126 140).

3. El precio cotizado según los costos de producción y distribución. En el caso que se viene explicando, este valor sería analizado considerando un margen del 30\% sobre los costos totales de \$132.31, lo que daría como resultado $\$ 172.00$ por cabina. De acuerdo a la sensibilidad de fluctuación del tipo de cambio, es importante monitorear este impacto. De ese modo, si en los próximos meses el tipo de cambio llega a los $\mathrm{S} / 3.60$ y se quiere mantener una utilidad del $30 \%$, sería posible colocar las cabinas a menor precio. Por otro lado, si el tipo de cambio baja a $S$ / 3.30, se tendría que vender el producto a mayor precio para mantener el margen de $30 \%$.

\section{CONCLUSIONES}

1. Los cambios en los procesos de importación y exportación originados por las estrategias de negocio modifican el comportamiento de los costos, lo que causa que los costos indirectos no atribuibles al costo de los bienes y servicios se conviertan en costos inventariables atribuibles.

2. Las estructuras de costos convencionales no son suficientes para determinar los costos asociados a la importación y exportación.

3. Las diferencias en los cambios monetarios impactan de forma diferente, según se tomen en cuenta los procesos de importación y exportación.

4. El reconocimiento de las operaciones de importación usualmente no están efectuadas de acuerdo a las fechas de su realización.

\section{RECOMENDACIONES}

1. Revisar constantemente el comportamiento de los costos y hacer que los costos indirectos no atribuibles al costo de los bienes y servicios se conviertan en costos inventariables atribuibles.

2. Formular estructuras de costos flexibles para poder determinar los costos asociados a la importación y exportación, ya sea en soles o en dólares.

3. Monitorear el impacto de las diferencias de cambios teniendo en cuentas los procesos de importación y exportación.

4. Identificar los rubros de operaciones monetarias y no monetarias para un adecuado tratamiento de conversión de moneda, especialmente, de aquellos costos locales en soles que tengan que convertirse en moneda extranjera.

5. El reconocimiento de las operaciones de importación debe ser efectuado de acuerdo a las fechas de la realización de estas actividades y según sistemas de registro en Bimoneda. 


\section{REFERENCIAS BIBLIOGRÁFICAS}

1. DANIELS, J., RADEBAUGH, L. \& SULLIVAN, D. (2004). Negocios internacionales. Décima edición. México: Pearson Educación.

2. HORNGREN, Ch., FOSTER, G. \& DATAR, S. (2012). Contabilidad de costos. Un enfoque gerencial. Decimoquinta edición. México: Prentice-Hall Hispanoamericana S.A.

3. INEI. (2 de febrero de 2016). Boletín técnico. Archivo en línea recuperado de: https://www. inei.gob.pe/media/MenuRecursivo/boletines/ informe-tecnico-n02_exportaciones-e-importacionesdic2015.pdf

4. Ministerio de Economía y Finanzas del Perú. (2016). NIC 21. Efectos de las Variaciones en las Tasas de Cambio de la Moneda extranjera. Archivo en línea recuperado de: http://www.mef. gob.pe/contenidos/conta_publ/con_nor_co/ no_oficializ/ES_GVT_IAS21_2015.pdf

5. Ministerio de Economía y Finanzas del Perú. (2016). Norma Internacional de Contabilidad 2. NIC 2. Inventarios. Archivo en línea recuperado de: http://www.mef.gob.pe/contenidos/conta_publ/con_nor_co/no_oficializ/ ES_GVT_IAS02_2015.pdf

6. SUNAT. (s/f). Drawback web. Registro en el portal web Sunat. Archivo en línea recuperado de: http://www.siicex.gob.pe/siicex/resources/ capacitacion/2014-7Drawback\%20Web\%20 -\%20SUNAT\%201.pdf 